\title{
Integral Field Spectroscopy of Selected Extragalactic Objects with FUEGOS
}

\author{
Jean Surdej ${ }^{1}$, Christian Vanderriest ${ }^{2}$, Marie-Christine Angonin-Willaime ${ }^{3}$, Jean- \\ François Claeskens ${ }^{4}$, Paul Felenbok ${ }^{3}$ \\ 1 Space Telescope Science Institute ${ }^{2}$, 3700 San Martin Drive, Baltimore, MD 21218, \\ USA \\ 2 Canada-France-Hawaii Telescope Corporation ${ }^{3}$, P.O. Box 1597, Kamuela, HI 96743, \\ USA \\ 3 Observatoire de Meudon, Place Jules Janssen, F-92195 Meudon Cedex, France \\ 4 European Southern Observatory, La Silla, Casilla 19001, Santiago 19, Chile
}

\begin{abstract}
Combining the large collecting area of the VLT, optimal seeing conditions (typically < 0.7 ") and the integral field spectroscopic capabilities (ARGUS mode) of FUEGOS (also known as the Multi-Fibre Area Spectrograph), it will become possible to address several new and important scientific issues in the field of gravitational lensing, physical studies of quasar host galaxies, etc. After a brief introduction, we recall some of the well known effects caused by macro- and micro- lensing as well as a few related applications of great astrophysical and cosmological interest. We then review the expected instrumental performance of FUEGOS in its ARGUS mode and the type of observational data that can be retrieved. Finally, we describe typical challenging FUEGOS/ARGUS observations of gravitational lens systems that could be successfully carried out at the Nasmyth focus of VLT No 3.
\end{abstract}

\section{Introduction}

It is well known that superb direct imagery and spectroscopic observations can be achieved, under seeing conditions as good as 0.5 ", with telescopes like the CFH $3.6 \mathrm{~m}$ in Hawaii, and also the ESO/MPI 2.2m and the NTT at La Silla. Seeing monitor campaigns carried out at ESO, and also elsewhere, have confirmed the occurence of such unusual conditions. The atmospheric seeing in Paranal is known to remain better than 0.5 " during approximately $16 \%$ of the clear nights (periods of at least one hour; see Sarazin 1990). Furthermore, the median seeing over the last years in Paranal has been measured to be 0.66 "; with an exceptional average seeing of 0.32 ", recorded during one full, best night. Therefore, astronomers should be given the technical possibility to obtain with the VLT, under such very good seeing conditions (FWHM $<0.66$ "), first class scientific

\footnotetext{
${ }^{2}$ Member of the Astrophysics Division, Space Science Department of the European Space Agency; on leave from the Fonds National de la Recherche Scientifique, Institut d'Astrophysique de l'Université de Liège, Belgium

${ }^{3}$ on leave from the Observatoire de Meudon, France
} 
observations of faint extragalactic objects (not only direct imagery but also spectroscopy), with optimal angular resolution sampling. In subsequent sections, we shall describe several extragalactic projects, including observational studies of selected gravitational lens systems for which major scientific achievements could be made thanks to the large collecting area of the VLT combined with a high angular resolution, bi-dimensional, spectroscopic mode. Let us first recall some of the basic features of gravitational lensing.

\section{Macro- and Micro-Lensing Effects}

Because of the deformation of the space time near a massive object (e.g. a galaxy lens), we know that a spherical wavefront originating from a distant source (cf. a quasar) will get distorted in the vicinity of the galaxy and that if an observer happens to lie very close to the line connecting the source and the lens, the former will see multiple images of the background quasar. For a typical galaxy deflector and cosmological redshifts for the source (QSO) and the lens, angular separations between the lensed images are typically of the order of 1 or several arcsec (see Refsdal and Surdej, 1994 for a recent review on gravitational lensing). We also know that because the light trajectories have different geometrical lengths, and also because time retardation varies along the different trajectories, a time delay $\Delta t$ will become measurable between the lensed images by the observer, provided of course that the quasar source undergoes photometric light variations. We know from the work of Refsdal (1964) that $\Delta t$ is inversely proportional to $H_{o}$, and it is therefore straightforward (at least in principle) to derive $H_{o}$ from $\Delta t$ if we dispose of a good model for the lens. Such a good lens model may be derived if we are able to measure the source redshift, the lens redshift, the positions of the lensed images with respect to that of the deflector, their flux ratio(s) (via the ratio(s) of emission-line fluxes, see below), the velocity dispersion of the deflector and, if possible, the mapping of that velocity dispersion across the deflector (cf. Falco et al. 1991 for the particular modelling of $0957+561 \mathrm{~A}$ and B).

There is however one more difficulty. When a foreground galaxy (the "macrolens") produces multiple images of a background quasar, these images are seen through rather dense parts of the galaxy and there is a good chance that one or several "macro-images" are affected by micro-lensing (Chang and Refsdal 1979). The "micro-lens" is a star (or several stars) of the galaxy, acting as a magnifying lens with a very small "field of view", which produces a more or less intricated network of micro-caustics (cf. Kayser 1992 and Wambsganss 1993 for recent reviews on micro-lensing). When the source crosses this network, its different components are differentially amplified, according to their sizes and locations. There will thus result a differential amplification of the different components of the quasar. For instance, in the spectrum of a micro-lensed quasar image, the optical continuum will be more amplified than the Broad Line Region (BLR) which has a larger extension. Due to relative proper motions, this phenomenon varies on a time scale of a few months or years and produces characteristic light curves (and very likely slightly variable spectroscopic line profiles for the 
broad emission-lines). Of course, the shape of these curves depends on the size of the source. A spectroscopic monitoring of the lensed QSO images will thus allow the structure and size of the continuum source to be probed, as well as the distribution in size (with an angular resolution of the order of $10^{-6 "}$ ) and velocity of the BLR clouds.

High resolution spectroscopy of the multiple (2-4) lensed images of a background quasar should also allow one to set interesting constraints on the size and structure of the Ly- $\alpha$ and/or heavy absorbing element clouds located along their lines-of-sight (cf. Smette et al. 1992). It should even be possible from such observations to set constraints on the deceleration parameter $q_{0}$ of the Universe.

So we see that gravitational lens systems, consisting of several variable macrolensed images and of a deflector with angular separations of the order of one second of arc, constitute unique laboratories to probe very important cosmological and astrophysical parameters.

\section{Description of the ARGUS Mode of FUEGOS}

One of the astronomer's dreams would of course be to obtain individual spectra for all contiguous elements covering the (very narrow angular) field of compact gravitational lens systems, or fuzz around distant quasars, etc. It will in fact be possible to achieve such observations in the ARGUS mode of the spectrograph FUEGOS which should normally be installed at the Nasmyth focus of VLT No 3 (see Felenbok et al. 1994 and Felenbok 1994, in these proceedings, for a complete and detailed description of this instrument). We should like to stress here that somewhat similar instruments (cf. SILFID described in Vanderriest and Lemonnier 1988, TIGER in Courtès et al. 1988, ARGUS of MOS at CFH in Vanderriest, 1994) have already been used successfully at the focus of $4 \mathrm{~m}$ telescopes.

In the FUEGOS spectrograph, 670 optical fibres forming the ARGUS bundle (this is the maximum number of fibres that can be accommodated across the 2048 $\times 2048$ CCD format; pixel size of 24 microns) are closely packed together on the axis of the telescope focus to provide a good spatial sampling for small extended objects (cf. Fig. 3 in Felenbok 1994, these proceedings). The ARGUS fibre tips are provided with the same type of micro-lens array (array of hexagonal microlenses) as in the MEDUSA design. At the input extremity, they optimise the field aperture filling factor, while at the other end they transform the fast fibre output beam for the $\mathrm{f} / 10$ spectrograph collimator. To satisfy the scientific requirements, three different spatial samplings are provided by means of three optical relays, which can be selectively positioned in front of the ARGUS entrance (angular field of 5 " $\times 5$ " with 0.2 " sampling, or 12 " $\times 12$ " with 0.45 " sampling, or 18 " $\times$ 18 " with 0.7 " sampling). As in the MEDUSA mode, spectral resolutions of $\mathrm{R}=$ 1500 (covering a range of $3500 \AA$ ) $, R=5000, R=10000$ and $R=30000$ will be accessible in the spectral range $3700-9000 \AA$. The ARGUS mode is provided with an atmospheric dispersion corrector $(\mathrm{ADC})$ which can be inserted, when necessary, in the incoming telescope beam. 
Of course, observations of gravitational lenses in this mode should only be carried out under excellent seeing conditions, i.e. in a service observing mode. Handling of the recorded spectra will make it possible to reconstruct images, by inverse geometrical transformation, in the QSO source continuum at any chosen wavelength - or range of wavelengths -, in specific emission- lines, to measure the velocity dispersion of the deflector, to produce maps of the radial velocity dispersion across the lens, maps of the radial velocity, of emission-line ratios, etc. (cf. Adam et al. 1989, Fitte and Adam 1993). Further analysis of these images should enable one to decompose the observed mirage into its individual lensed images plus deflecting galaxy by adequate fitting techniques.

\section{FUEGOS/ARGUS Observations of Selected Extragalactic Objects}

\subsection{Gravitational Lens Systems}

For most of the presently known gravitational lens systems, the distant $\left(z_{L}>\right.$ 0.5 ) deflecting galaxies are generally much fainter $\left(R_{L} \simeq 19-23\right)$ than the lensed QSO images. The information available on most of these lenses is thus dramatically poor. Two-dimensional spectroscopy will allow optimal separation of the faint light of the lens from the glare of the lensed quasar images. It will be possible to sum up the signal from all the fibres illuminated by the galaxy and subtract the quasar contamination. Let us note that thanks to the 'image slicer' effect, the spectral resolution will be kept constant for each individual image. We would recommend to derive $(D)$ or to confirm $(C)$ the lens redshift $z_{L}$ and/or to measure the velocity dispersion $(V D)$ for the following lens galaxies (see Angonin-Willaime et al. 1993 and Refsdal and Surdej 1994 for more information on the individual objects): UM673 $\left(R_{L} \simeq 19\right.$ and $z_{L} \simeq 0.49, C$, $V D), \mathrm{PG} 1115+080\left(R_{L} \simeq 19.8\right.$ and $\left.z_{L} \simeq 0.29, C, V D\right), 2016+112\left(i_{L} \simeq 22\right.$ and $z_{L} \simeq 1.01$ and 2 ?, $D$ and $\left.C\right)$, MG0414+0534 ( $R_{L} \simeq 22.4$ and $z_{L} \simeq 0.47, C$, $V D), \mathrm{PKS1830-211}\left(V_{L} \simeq 23, D\right), \mathrm{B} 0218+357\left(r_{L} \simeq 20\right.$ and $\left.z_{L} \simeq 0.68, C, V D\right)$, $2237+0305\left(R_{L} \simeq 14.5\right.$ and $z_{L} \simeq 0.04$, mapping of the $\left.V D\right), 1654+1346\left(r_{L} \simeq\right.$ 18.7 and $z_{L} \simeq 0.25, \mathrm{C}$ and $V D$ ). In order to have an approximate idea about the requested exposure times, we have estimated for the case of PG1115+080 that, using a spatial sampling of 0.4 " per pixel and a spectral resolution $R=1500$, it should be possible to obtain in 5 hours a good spectrum for the lensing galaxy characterized by a signal-to-noise ratio $\mathrm{S} / \mathrm{N} \simeq 20$. From the same exposure, it will also be possible to extract individual spectra for the 3 lensed QSO images ( $\mathrm{A}, \mathrm{B}$ and $\mathrm{C})$, characterized by a $\mathrm{S} / \mathrm{N} \simeq 100-500$, depending on their apparent brightness. Using a spatial sampling of 0.2 " per pixel, it should even be possible to record in just 2 hours individual spectra $(S / N \simeq 200)$ for the two merging macro-images A1 and A2, separated by only 0.5 ".

In addition, very high signal-to-noise and high resolution spectra (with a sampling of 0.2" per pixel) of the individual lensed QSO images for UM673, PG1115+080, 2237+0305, H1413+117 (see Angonin et al. 1990 for similar observations obtained with SILFID at CFH, sampling of 0.33 " per pixel), B1422+231, 
1009-025, HE1104-1805, etc. will allow the structure and size of the continuum source to be probed, as well as the distribution in size and velocity of the BLR clouds (see discussion in the previous section). These data will also be used to constrain the transversal size of (Ly- $\alpha$ and metallic) absorption line clouds (cf. Smette et ail. 1992).

\subsection{Other Extragalactic Objects}

On account of space limitations, we shall only enumerate hereafter additional types of faint extragalactic objects for which 2-D spectroscopic observations with FUEGOS would also be very useful:

i. bright giant luminous arcs such as A370, Cl2244-02 (mapping the velocity dispersion across the lensed galaxies)

ii. the galaxies detected near (i.e. < 3") Highly Luminous Quasars (redshift and velocity dispersion measurements of those galaxies $(20 \lesssim R \lesssim 23$ ) to independently estimate the magnification bias and to test our understanding on the cosmic distribution, luminosity function and evolution of galaxies in the Universe)

iii. the faint $(R>21)$ environment of distant luminous quasars (extended haloes?, cooling flows in a surrounding cluster?)

iv. the host galaxies around low redshift quasars

v. kinematics (2-D velocity field) of the central regions of galaxies

vi. resolved structures in the inner regions of AGNs and merging galaxies (mapping velocities, excitation parameters, abundances...)

vii. etc.

\section{References}

Adam, G., Bacon, R., Courtès, G., Georgelin, Y., Monnet, G., Pécontal, E.: 1989, Astron. Astrophys. 208, L15

Angonin, M.-C., Remy, M., Surdej, J., Vanderriest, C.: 1990, Astron. Astrophys. 233, L5

Angonin-Willaime, M.-C., Hammer, F., Rigaut, F.: 1993, review paper in the proceedings of the $31^{\text {st }}$ Liège International Astrophysical Colloquium 'Gravitational Lenses in the Universe', p. 85, Eds. Surdej, J., Fraipont-Caro, D., Gosset, E., Refsdal, S. and Remy, M. (University of Liège, Belgium)

Chang, K., Refsdal, S.: 1979, Nature 282, 561

Courtès, G., Georgelin, Y., Bacon, R., Monnet, G., Boulesteix, J.: 1988, in the proceedings of 'Instrumentation for Ground-Based Optical Astronomy: Present and Future', p. 266, Ed. Robinson, L.B.

Falco, E.E., Gorenstein, M.V., Shapiro, I.I.: 1991, Astrophys. J. 372, 364

Felenbok, P., Cuby, J.-G., Lemonnier, J.-P., Baudrand, J., Casse, M., Andre, M., Czarny, J., Daban, J.-B., Marteaud, M., Vola, P.: 1994, SPIE: Instrumentation in Astronomy V III, 2198, 115

Felenbok, P.: 1994, these proceedings 
Fitte, C., Adam, G.: 1993, in the proceedings of the $31^{\text {st }}$ Liège International Astrophysical Colloquium 'Gravitational Lenses in the Universe', p. 445, Eds. Surdej, J., Fraipont-Caro, D., Gosset, E., Refsdal, S. and Remy, M. (University of Liège, Belgium)

Kayser, R.: 1992, in 'Gravitational Lenses', (Proceedings, Hamburg 1991), Lecture Notes in Physics 406, p. 143

Refsdal, S.: 1964, Monthly Notices Roy. Astron. Soc. 128, 307

Refsdal, S., Surdej, J.: 1994, Reports on Progress in Phys. 57, 117

Sarazin, M.: 1990, ESO-VLT Report N0 62

Smette, A., Surdej, J., Shaver, P.A., Foltz, C.B., Chaffee, F.H. et al.: 1992, Astrophys. J. $\mathbf{3 8 9}, 39$

Vanderriest, C., Lemonnier, J.-P.: 1988, in the proceedings of 'Instrumentation for Ground-Based Optical Astronomy: Present and Future', p. 304, Ed. Robinson, L.B. Vanderriest, C.: 1994, CFH Information Bulletin, 31, 22.

Wambsganss, J.: 1993, review paper in the proceedings of the $31^{\text {st }}$ Liège International Astrophysical Colloquium 'Gravitational Lenses in the Universe', p. 369, Eds. Surdej, J., Fraipont-Caro, D., Gosset, E., Refsdal, S. and Remy, M. (University of Liège, Belgium) 